Old Dominion University

ODU Digital Commons

Physics Faculty Publications

Physics

$8-2018$

\title{
Compensation of Non-Linear Bandwidth Broadening by Laser Chirping in Thomson Sources
}

C. Maroli

V. Petrillo

I. Drebot

L, Serafini

B. Terzić

Old Dominion University, bterzic@odu.edu

See next page for additional authors

Follow this and additional works at: https://digitalcommons.odu.edu/physics_fac_pubs

Part of the Atomic, Molecular and Optical Physics Commons, and the Optics Commons

\section{Repository Citation}

Maroli, C.; Petrillo, V.; Drebot, I.; Serafini, L,; Terzić, B.; and Krafft, G. A., "Compensation of Non-Linear Bandwidth Broadening by Laser Chirping in Thomson Sources" (2018). Physics Faculty Publications. 242.

https://digitalcommons.odu.edu/physics_fac_pubs/242

\section{Original Publication Citation}

Maroli, C., Petrillo, V., Drebot, I., Serafini, L., Terzić, B., \& Krafft, G. A. (2018). Compensation of non-linear bandwidth broadening by laser chirping in Thomson sources. Journal of Applied Physics, 124(6), 063105. doi: 10.1063/1.5033549

This Article is brought to you for free and open access by the Physics at ODU Digital Commons. It has been accepted for inclusion in Physics Faculty Publications by an authorized administrator of ODU Digital Commons. For more information, please contact digitalcommons@odu.edu. 
Authors

C. Maroli; V. Petrillo; I. Drebot; L, Serafini; B. Terzić; and G. A. Krafft 


\section{Compensation of non-linear bandwidth broadening by laser chirping in Thomson sources}

C. Maroli, V. Petrillo, I. Drebot, L. Serafini, B. Terzić, and G. A. Krafft

Citation: Journal of Applied Physics 124, 063105 (2018); doi: 10.1063/1.5033549

View online: https://doi.org/10.1063/1.5033549

View Table of Contents: http://aip.scitation.org/toc/jap/124/6

Published by the American Institute of Physics

\section{Articles you may be interested in}

Incoherent control of optical bistability and multistability in a hybrid system: Metallic nanoparticle-quantum dot nanostructure

Journal of Applied Physics 124, 063102 (2018); 10.1063/1.5038874

A self-activated $\mathrm{MgGa}_{2} \mathrm{O}_{4}$ for persistent luminescence phosphor Journal of Applied Physics 124, 063101 (2018); 10.1063/1.5024771

Dual-band electromagnetically induced transparency effect in a concentrically coupled asymmetric terahertz metamaterial

Journal of Applied Physics 124, 063106 (2018); 10.1063/1.5040734

Dynamic behavior of $\mathrm{CH}_{3} \mathrm{NH}_{3} \mathrm{Pbl} 3$ perovskite twin domains

Applied Physics Letters 113, 072102 (2018); 10.1063/1.5041256

Interplay of cation ordering and thermoelastic properties of spinel structure $\mathrm{MgGa}_{2} \mathrm{O}_{4}$

Journal of Applied Physics 124, 065111 (2018); 10.1063/1.5037786

Imaging in-plane $90^{\circ}$ magnetization switching in a (Ga,Mn)As epitaxial layer

Journal of Applied Physics 124, 063901 (2018); 10.1063/1.5040129

\section{AIP| $\left.\right|_{\text {Aaphal of }} ^{\text {souna }}$ Applied Physics SPECIAL TOPICS}

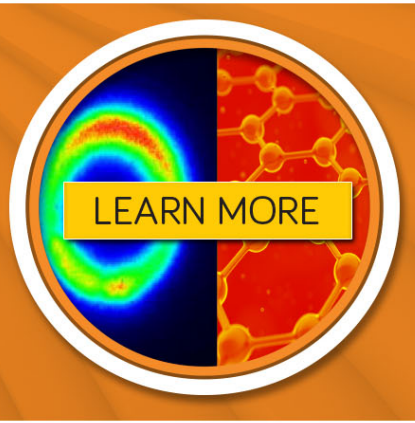




\title{
Compensation of non-linear bandwidth broadening by laser chirping in Thomson sources
}

\author{
C. Maroli, ${ }^{1}$ V. Petrillo, ${ }^{1,2}$ I. Drebot, ${ }^{2}$ L. Serafini, ${ }^{2}$ B. Terzić, ${ }^{3}$ and G. A. Krafft ${ }^{3,4}$ \\ ${ }^{1}$ Università degli Studi di Milano, via Celoria 16, 20133 Milano, Italy \\ ${ }^{2}$ INFN-Sezione di Milano, via Celoria 16, 20133 Milano, Italy \\ ${ }^{3}$ Department of Physics, Center for Accelerator Science, Old Dominion University, Norfolk, \\ Virginia 23529, USA \\ ${ }^{4}$ Thomas Jefferson National Accelerator Facility, Newport News, Virginia 23606, USA
}

(Received 6 April 2018; accepted 21 July 2018; published online 10 August 2018)

\begin{abstract}
A new laser chirping prescription is derived by means of the phase-stationary method for an incident Gaussian laser pulse in conjunction with a Liénard-Wiechert calculation of the scattered radiation flux and spectral brilliance. This particularly efficient laser chirp has been obtained using the electric field of the laser and for electrons and radiation on axis. The frequency modulation is somewhat reduced with respect to that proposed in the previous literature, allowing the application of this procedure to lasers with larger values of the parameter $a_{0}$. Numerical calculations have been performed using mildly focused and narrow bandwidth laser pulses, confirming a larger efficiency of the chirp prescription here introduced. The chirp efficiency has been analysed as a function of the laser parameter and focusing. Published by AIP Publishing. https://doi.org/10.1063/1.5033549
\end{abstract}

\section{INTRODUCTION}

$\mathrm{X}$ - and gamma-ray sources with large spectral flux and high tunability allow one to understand the properties of materials and living systems by probing the matter on microscopic-to-atomic and nuclear scales in space and time., Thomson $^{3-13}$ sources are among the best performing devices to produce $\mathrm{x}$ radiation with narrow bandwidth, high power, and wide tunability, from accelerators with relatively small dimensions and costs. Experiments on the source characterization, ${ }^{14-17}$ imaging, K-edge techniques, and computed microtomography on phantom, ${ }^{18,19}$ biological, ${ }^{6,15}$ animal, ${ }^{11,20,21}$ and human ${ }^{7,11}$ samples with $\mathrm{keV}$ range $\mathrm{x}$-rays have been already successfully performed. On the other hand, inverse Compton sources, such as the facility Higs ${ }^{22}$ or the advanced Gamma System ELI-NP-GS, ${ }^{23,24}$ are aimed at producing extreme gamma ray beams for nuclear physics and nuclear photonics experiments. In these fields, extremely narrow relative bandwidths $\Delta \omega / \omega$, down to few $10^{-3}$, are required.

Achieving large spectral fluxes with narrow bandwidths is one of the most demanding challenges in the Thomson/ Compton physics. In the linear regime, occurring when the laser parameter $a_{0}=e E_{0} /\left(m c \omega_{L}\right)$ is much smaller than one ( $E_{0}$ and $\omega_{L}$ being, respectively, the peak electric field and the angular frequency of the laser pulse), the relative bandwidth of the radiation of one single electron collected on axis reflects the relative bandwidth of the laser $\Delta \omega_{L} / \omega_{L}$. Starting from this minimum value, many factors contribute to enlarge the bandwidth. First of all, the frequency-angle correlation constitutes an unavoidable limitation connected with the necessity of gathering the radiation within a given acceptance angle. Furthermore, considering the emission from a real electron beam, emittance and energy spread of the particles must be taken carefully under control, in order to diminish their negative effects. ${ }^{25-27}$ Another source of bandwidth broadening is the non-linear ponderomotive effect that takes place when $a_{0}$ is of the order or larger than $1 .^{28-30}$ The advantage of increasing the luminosity of the source by enhancing the primary photon number is in fact strongly limited by the intrusion in the spectrum of multiple secondary peaks, ${ }^{31-36}$ due to the interference between wave trains emitted from different positions within the laser pulse. Their number has been empirically estimated as about $N$ $=\omega_{L} a_{0}^{2} \Delta t / 4 \sqrt{2 \pi}$ ( $\Delta t$ is the laser pulse duration). ${ }^{33,37} \mathrm{In}$ addition, when $a_{0}$ increases, the maximum spectral density on the fundamental peak shifts towards lower frequency values, according to $\omega_{\text {peak }}=4 \omega_{L} \gamma_{0}^{2} /\left(1+a_{0}^{2} / 2\right)$, and the spectrum fills the entire region between $\omega_{\text {peak }}$ and the linear edge $4 \omega_{L} \gamma_{0}^{2}, \gamma_{0}$ being the initial Lorentz factor of the electron. In Refs. 37 and 38, a very powerful method for compensating the non-linear bandwidth broadening has been presented. They demonstrated that the presence of a calibrated temporal frequency modulation of the laser-a laser chirp-corrects almost completely the enlargement of the radiation bandwidth due to scattering with a single electron on axis, impeding the formation of oscillations. The physical mechanism is the following: when entering the laser pulse, the electron experiences a varying laser parameter and emits radiation whose frequency varies during the emission process, due to its dependence on the instantaneous electron velocity, on the local laser parameter and on the laser frequency. A temporal variation of the laser frequency along the pulse balances the temporal variation of the other quantities and restores the monochromaticity. In Refs. 37-39, chirp shapes have been calculated and tested, showing a compensation of the nonlinear broadening up to laser parameter values close to 1 . The same procedure has been advantageously extended to the harmonics ${ }^{40}$ or to circular polarization. ${ }^{41}$ Seipt et al. ${ }^{42}$ proposed a quantum treatment of the problem based on Volkov states, demonstrating that the best chirp does not 
depend on quantum recoil and arriving to a chirp prescription similar to that of Ref. 37.

In this paper, we give a laser chirp profile which extends that derived in Ref. 37 in the case of large $a_{0}$. The derivation has been done by applying the stationary phase method to the double differential spectrum emitted by one single electron in head-on collision with a Gaussian laser field. The chirp has been calculated for electrons moving and radiation emitted on axis, in the limit of large laser waist. Realistic situations are then analyzed numerically, by adopting a mildly focused and narrow bandwidth laser and collecting the chirped radiation within given acceptance angles $\theta_{\max }$, thus evaluating the integrated spectrum. The variation of the laser waist $w_{0}$ is analyzed. A reduction of the bandwidth from the linear value $(\triangle \omega / \omega) \approx \sqrt{\left(\triangle \omega_{L} / \omega_{L}\right)^{2}+\left(\gamma_{0} \theta_{\max }\right)^{4} / 12}$ to the limiting value $\Delta \omega_{L} / \omega_{L}$ with increasing $a_{0}$ is furthermore shown.

\section{CHIRP CALCULATION}

The calculation has been done in the classical regime, where the quantum recoil is negligible, i.e., when $\hbar \omega_{L} \gamma_{0} / m c^{2} \ll 1$. We consider as the primary photon source a Gaussian laser pulse with polarization parallel to the $\mathrm{X}$-axis, moving along the negative $\mathrm{Z}$-axis with velocity $c$. Differently from the previous literature, mainly based on the use of the vector potential, we start from the electric field of the pulse, which is given by

$$
\underline{E}(x, y, z, t)=\underline{e}_{x} E_{0}\left(\sin \Gamma-\frac{z}{z_{R}} \cos \Gamma\right) \mathcal{G},
$$

where

$$
\mathcal{G}=\frac{1}{1+\frac{z^{2}}{z_{R}^{2}}} \exp \left(-\frac{\zeta^{2}}{2 \sigma_{\|}^{2}}-\frac{x^{2}+y^{2}}{2 \sigma_{\perp}^{2}\left(1+z^{2} / z_{R}^{2}\right)}\right) .
$$

Here $\zeta=z+c t, z_{R}=k_{L} \sigma_{\perp}^{2}$ is the Rayleigh length, $\sigma_{\|}$and $\sigma_{\perp}$ are the rms longitudinal and transverse dimension of the modulation and $k_{L}=2 \pi / \lambda_{L}$ the carrier wave-number. The function $\Gamma$ in Eq. (1) is

$$
\Gamma=k_{L} f(\zeta) \zeta+\frac{z z_{R}\left(x^{2}+y^{2}\right)}{2 \sigma_{\perp}^{2}\left(z^{2}+z_{R}^{2}\right)},
$$

where

$$
f(\zeta)=\frac{\omega(\zeta)}{\omega_{L}}=\frac{k(\zeta)}{k_{L}}
$$

is the frequency modulation giving the local value of the angular frequency $\omega(\zeta)$ [or wave-number $k(\zeta)$ ] normalized by the carrier frequency $\omega_{L}$ (or wave-number $k_{L}$ ). For a laser pulse without chirp, $f=1$. Equation (1) is an approximation of the electric field of the laser pulse solution of the Maxwell equation, in the limit of small $1 /\left(\sqrt{2} \sigma_{\perp} k_{L}\right)$ and $1 /\left(\sqrt{2} \sigma_{\|} k_{L}\right)$ and if all modulations are slowly varying functions (the paraxial approximation ${ }^{43}$ ).

By the same argument, the chirp $f(\zeta)$ must also be a slowly varying function of the variable $\zeta$.
For calculating the radiation, we considered the double differential energy spectrum as given in Ref. 44

$$
\frac{d^{2} W}{d \omega d \Omega}=\frac{e^{2}}{4 \pi^{2} c}\left|\int_{-\infty}^{\infty} d t \mathrm{e}^{i \omega \mathrm{t}}\left(\frac{\underline{n} \times((\underline{n}-\underline{\beta}) \times \underline{\hat{\beta}}}{(1-\underline{n} \cdot \underline{\beta})^{3}}\right)_{r e t}\right|^{2} .
$$

The laser field experienced by an electron moving close to the axis and in the limit of very large $\sigma_{\perp}$ reduces to

$$
\underline{E}(x, y, z, t)=\underline{e}_{x} E_{0} \sin \left(k_{L} f(\zeta) \zeta\right) \exp \left(-\frac{\zeta^{2}}{2 \sigma_{\|}^{2}}\right)=\underline{e}_{x} E_{0} g(\zeta)
$$

the same expression used in the pulsed plane wave model.

The chirp prescription will be calculated on axis.

The electron motion equations in terms of the phase $\varphi\left(t^{\prime}\right)=k_{L}\left(z\left(t^{\prime}\right)+c\left(t^{\prime}-t_{0}\right)\right)\left(\mathrm{t}^{\prime}\right.$ retarded time) can be written as

$$
\begin{aligned}
& \frac{d p_{x}}{d \varphi}=-a_{0} g(\varphi), \\
& \frac{d p_{z}}{d \varphi}=a_{0} g(\varphi) \frac{p_{x}}{\gamma_{0}},
\end{aligned}
$$

with $a_{0}=e E_{0} /\left(\omega_{L} m c\right)$, leading to the relations

$$
p_{x}=-\int_{\varphi_{0}}^{\varphi} a_{0} g\left(\varphi^{\prime}\right) d \varphi^{\prime}
$$

$$
p_{z}=p_{z 0}-\frac{a_{0}^{2}}{2 \gamma_{0}}\left(\int_{\varphi_{0}}^{\varphi} g\left(\varphi^{\prime}\right) d \varphi^{\prime}\right)^{2}
$$

where $p_{z 0}$ and $\gamma_{0}$ are momentum and Lorentz factor, respectively, of the electron before the head-on collision with the laser pulse.

Calculating $\mathrm{t}^{\prime}$ and $z\left(\varphi^{\prime}\right)$ from (9) and (8) and inserting in (5), we can arrive to

$$
\begin{aligned}
\frac{d^{2} W}{d \omega d \Omega}= & \frac{e^{2} a_{0}^{2} \gamma_{0}^{2}\left(1+\beta_{z 0}\right)^{2}}{16 \pi^{2} c} \\
& \times\left|\sum_{n=1}^{\infty}(-)^{n} \int_{-\infty}^{\infty} d Y\left(J_{n}(\xi)+J_{n+1}(\xi)\right) e^{i \frac{\Phi_{n}}{\varepsilon}-Y^{2}}\right|^{2},
\end{aligned}
$$

where $\mathrm{n}$ is the harmonic index, $Y=\varepsilon k_{L} \zeta=(z+c t) /\left(\sqrt{2} \sigma_{\|}\right)$ ( $\mathrm{t}$ laboratory time), and $\beta_{z 0}$ is the velocity of the electron before the collision.

The argument $\xi$ of the Bessel function appearing in Eq. (10) is

$$
\xi=\frac{\bar{\omega} A(Y)}{2(\dot{b}(Y))^{3}},
$$

where $A(Y)=a_{0}^{2} \mathrm{e}^{-2 \mathrm{Y}^{2}} / 2$,

$$
\bar{\omega}=\frac{\omega}{\omega_{l i n}}=\frac{\omega}{\omega_{L} \gamma_{0}^{2}\left(1+\beta_{z 0}\right)^{2}},
$$

and $\dot{b}(Y)$ is the first derivative of the function $b(Y)=y f(Y)$, $f(Y)$ being the FM of the laser pulse as defined in (4). Finally, the phases $\Phi_{n}$ in (10) are 


$$
\Phi_{n}=\bar{\omega} Y+(1-2 n) b(Y)+\bar{\omega} \int_{0}^{y} d Y^{\prime} \frac{A\left(Y^{\prime}\right)}{\left(\dot{b}\left(Y^{\prime}\right)\right)^{2}} .
$$

By applying the stationary phase method to estimate the integral in 10 in the limit $\varepsilon \rightarrow 0$, as described in more details in Ref. 36, and equating to zero the first derivative of $\Phi_{n}$ with respect to $Y$ [the integration variable in Eq. (10)], we obtain

$$
\bar{\omega}=(2 n-1) \frac{(\dot{b})^{3}}{(\dot{b})^{2}+A(Y)} \quad(n=1,2,3 \ldots) .
$$

The customary procedure ${ }^{37,38}$ is to choose the chirp function $f(Y)$ in such a way that

$$
(\dot{b})^{3}=p\left((\dot{b})^{2}+A(Y)\right),
$$

where $p>0$ is an arbitrary constant. The electron is therefore forced to emit on the frequencies

$$
\omega_{n}=p(2 n-1) \omega_{\text {lin }} \quad(n=1,2,3 \ldots) .
$$

The solution of Eq. (15) is

$$
\dot{b}=\frac{d}{d Y}(Y f(Y))=s_{1}+s_{2}+\frac{p}{3},
$$

with

$$
s_{1,2}(Y)=\left[\frac{p}{2} A(Y)+\frac{p^{3}}{27} \pm \sqrt{\frac{p^{4}}{27} A(Y)+\frac{p^{2}}{4} A^{2}(Y)}\right]^{\frac{1}{3}} .
$$

Note that $s_{1}$ and $s_{2}$ are positive functions of $Y$ and therefore $\dot{b}(Y)$ is also larger than zero allowing the division by it in Eqs. (11), (13), and (14).

The only regular solution of the differential Eq. (17) is

$$
f(Y)=\frac{p}{3}+\frac{1}{Y} \int_{0}^{Y} d Y^{\prime}\left(s_{1}\left(Y^{\prime}\right)+s_{2}\left(Y^{\prime}\right)\right) .
$$

For small values of $a_{0}$, this function becomes

$$
f_{0}(Y)=p\left(1+\sqrt{\frac{\pi}{2}} \frac{a_{0}^{2}}{4 p^{2} Y} \operatorname{erf}(\sqrt{2} Y)\right),
$$

which is the frequency modulation first studied in Ref. 29, whose Eq. (6) is similar to our (17) provided that $z+c t$ $=\sqrt{2} Y \sigma(\sigma$ is the longitudinal laser dimension as defined in Ref. 29) and $p=1 /\left(1+a_{0}^{2} / 2\right)$, and then retrieved in Ref. 41 in different conditions of polarization. We consider the more general case (19) with $p=1$.

\section{NUMERICAL CALCULATIONS}

All the simulations have been done by calculating numerically the electron trajectory under the effect of the Gaussian laser pulse described by expression (1), representing a mildly focused and narrow-bandwidth laser. We have then computed the Liénard-Wiechert electromagnetic field

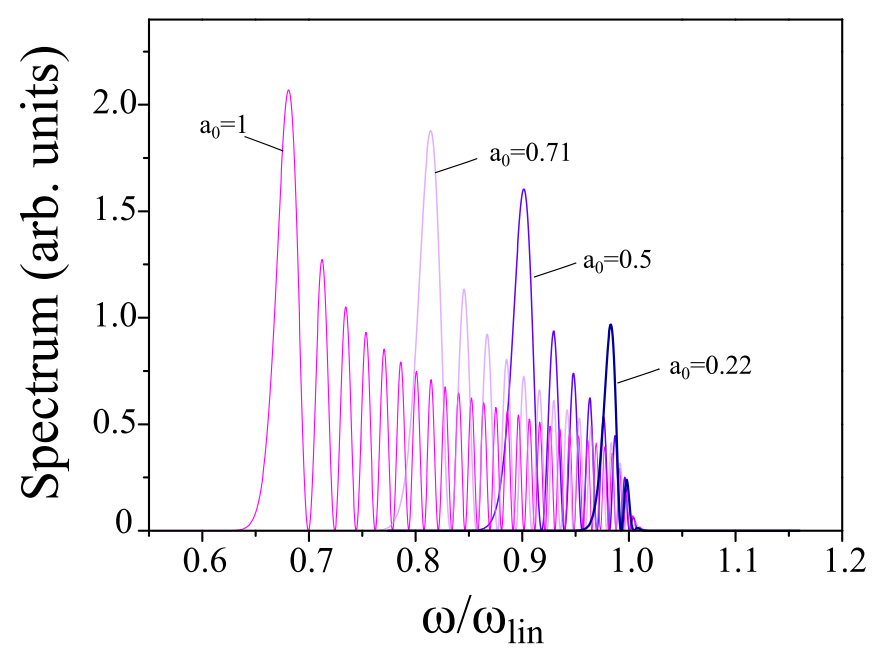

FIG. 1. Spectrum on axis in arbitrary units without chirp $(f=1)$, for $a_{0}$ $=0.22(E=0.1 \mathrm{~J}$, navy curve $), a_{0}=0.5$ ( $E=0.5 \mathrm{~J}$, violet curve), $a_{0}=0.71$ ( $E=1 \mathrm{~J}$, light magenta), and $a_{0}=1$ ( $E=2 \mathrm{~J}$, magenta curve). The number of peaks $N$, given by the exact formula in Ref. 37, is, respectively, $N=1.8$, 9.3, 18.7, and 37.5. The laser, with wavelength $\lambda_{L}=0.5 \mu \mathrm{m}$ and linearly polarized on the x-axis, has an rms time duration of $\Delta t=0.1 \mathrm{ps}$ and an rms spot diameter of $w_{0}=15 \mu \mathrm{m}$. The laser, with wavelength $\lambda_{L}=0.5 \mu \mathrm{m}$ and linearly polarized on the $\mathrm{x}$-axis, has an rms time duration of $\Delta t=0.1 \mathrm{ps}$ and an rms spot diameter of $w_{0}=15 \mu \mathrm{m}$.

radiated in the far region, as well as the energy spectrum detected on the screen.

In the numerical calculation, the laser, with wavelength $\lambda_{L}=0.5 \mu \mathrm{m}$ and linearly polarized on the $\mathrm{x}$-axis, has an rms time duration of $\Delta t=0.1 \mathrm{ps}$ and the electron started with an incident Lorentz factor value of $\gamma_{0}=60$. The shape of the spectrum of the radiation on axis for a laser without chirp $(f=1)$ is presented in Fig. 1 for an rms spot diameter of $w_{0}$ $=2 \sigma_{\perp}=15 \mu \mathrm{m}$ and various values of $a_{0}$, showing the development of the system of fringes.

Figure 2 shows the functions $f(Y)$ (red line) and $f_{0}(Y)$ (black line) as a function of $Y$, for $a_{0}=0.5$ and $a_{0}=1.58$. Note that Ref. 37 uses a different boundary condition for the frequency modulation function: $f(0)=1$. In the former case, $f(Y)$ and $f_{0}(Y)$ can hardly be distinguished on the scale of the figure; while enhancing $a_{0}$ they differ increasingly. A

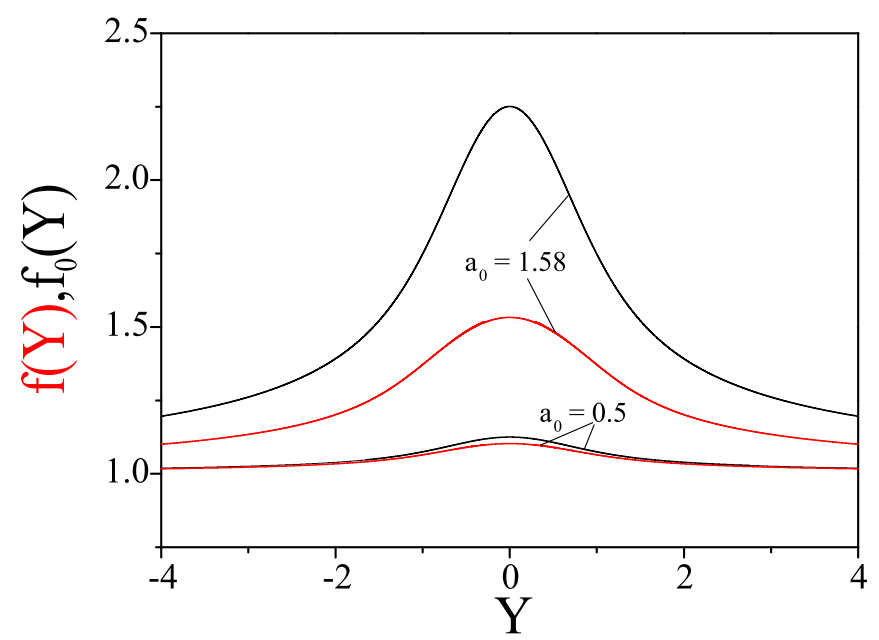

FIG. 2. Frequency modulation $f$ (red) and $f_{0}$ (black) as a function of their argument $Y=\varepsilon k_{L} \zeta=(z+c t) /\left(\sqrt{2} \sigma_{\|}\right)$for $a_{0}=0.5$ and $a_{0}=1.58$. 
substantial difference between Eq. (19) and all other chirp prescriptions proposed in the literature is the trend with $a_{0}$ in the limit of large $a_{0}$. In fact, the limit of Eq. (19) for $a_{0} \gg 1$ turns out to be

$$
f \approx a_{0}^{2 / 3}
$$

while all other chirp prescriptions follow the trend $a_{0}^{2}$.

This is connected to the use of the electric field in the motion equations. In fact, from (9), the longitudinal electron velocity can be evaluated approximately as

$$
\beta_{z} \approx \beta_{z 0}-\frac{a_{0}^{2}}{2 \gamma_{0}^{2} f^{2}},
$$

where $\int_{\varphi_{0}}^{\varphi} g\left(\varphi^{\prime}\right) d \varphi^{\prime}$ has been integrated by parts and calculated on the peak. Inserting (22) in the Doppler expression, we obtain

$$
\omega=\frac{\omega_{L} f\left(1+\beta_{z}\right)}{1-\beta_{z}} \approx \frac{2 \omega_{L} f}{1-\beta_{z 0}+\frac{a_{0}^{2}}{2 \gamma_{0}^{2} f^{2}}}=4 \gamma_{0}^{2} \omega_{L} \frac{f}{1+\frac{a_{0}^{2}}{f^{2}}} .
$$

The dependence $f \sim a_{0}^{2 / 3}$ for $a_{0}$ large can be then retrieved by equating

$$
\frac{f}{1+\frac{a_{0}^{2}}{f^{2}}}=1
$$

and results somewhat weaker with respect to the dependence on $a_{0}^{2}$.

Two cases, relevant to $a_{0}=0.5$ and $a_{0}=1$, are presented, respectively, in Figs. 3 and 4, showing the spectrum on axis in the far field.

In both figures, cases without chirp (in black) are compared with the same cases with the chirp given by $f_{0}$ (in red) and $f$ (in blue).

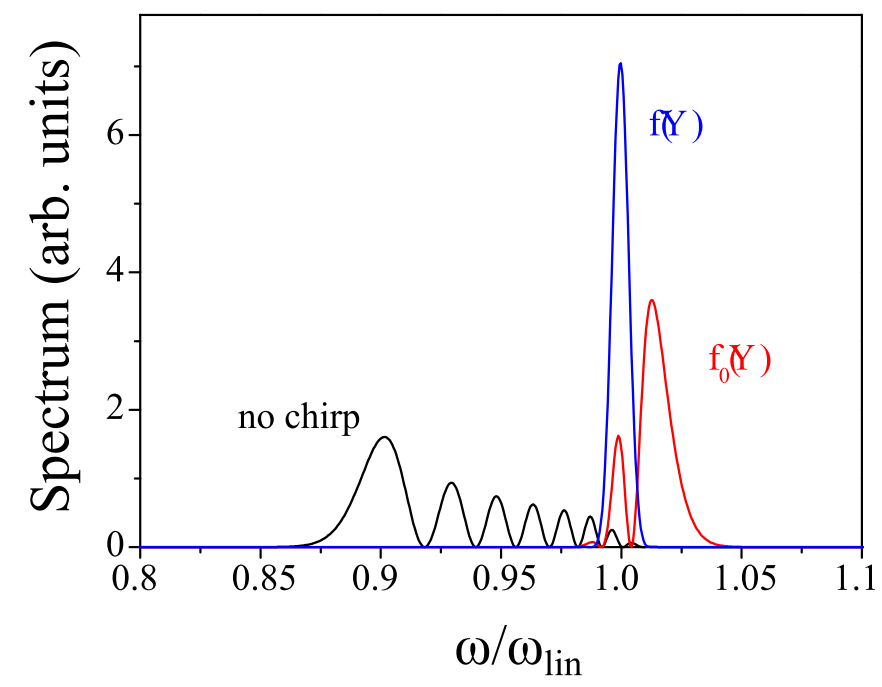

FIG. 3. Spectrum of the radiation on axis vs $\omega / \omega_{\text {lin }}$ for $a_{0}=0.5$. Black curve: no chirp, number of oscillations $N=9.3$. Red curve: chirp $f_{0}$. Blue curve: $\operatorname{chirp} f$.

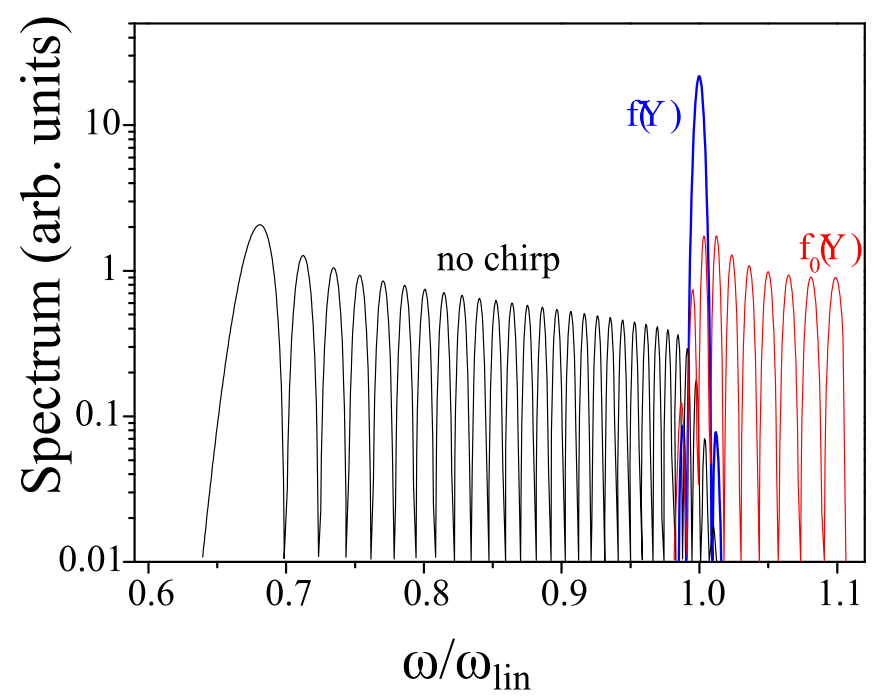

FIG. 4. Spectrum of the radiation on axis vs $\omega / \omega_{\text {lin }}$ for $a_{0}=1$. Black curve: no chirp, number of oscillations $N=37.5$. Red curve: chirp $f_{0}$. Blue curve: chirp $f$.

Without the chirp, the spectrum presents the wellknown oscillations, shown also in Fig. 1. The effect of the chirp is that of eliminating the ripples and shrinking the bandwidth. When $a_{0}=0.5$, (Fig. 3) the two expressions of the chirp give almost the same result, but for $a_{0}=1$ (Fig. 4), the expression $f_{0}$ hardly compensates the nonlinear broadening of the spectrum, while $f$ is amply efficient. The chirp $f$ is able to make monochromatic also radiation produced by high intensity lasers (with for instance $a_{0}=10$ ), as shown in Fig. 5 .

Since the detector or the sample in an experiment receive the radiation integrated over a surface, we have analyzed the spectrum of the signal collected within a solid acceptance angle (Figs. 6 and 7).

Figures 6(a) and 7(a) show the spectrum for an acceptance angle $\theta_{\max }=2 \mathrm{mrad}$ and $\theta_{\max }=4 \mathrm{mrad}$ for various values of $a_{0}$ without chirp. For the cases with larger $a_{0}$, the radiation on the harmonics produces the tails at the right.

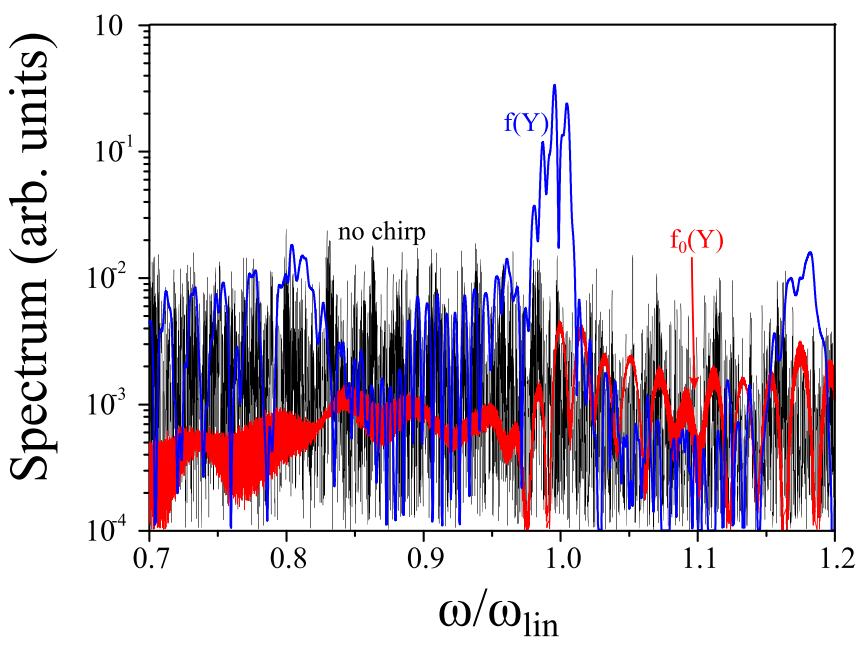

FIG. 5. Spectrum of the radiation on axis vs $\omega / \omega_{\text {lin }}$ for $a_{0}=10$. Black curve: no chirp. Red curve: $\operatorname{chirp} f_{0}$. Blue curve: $\operatorname{chirp} f$. 

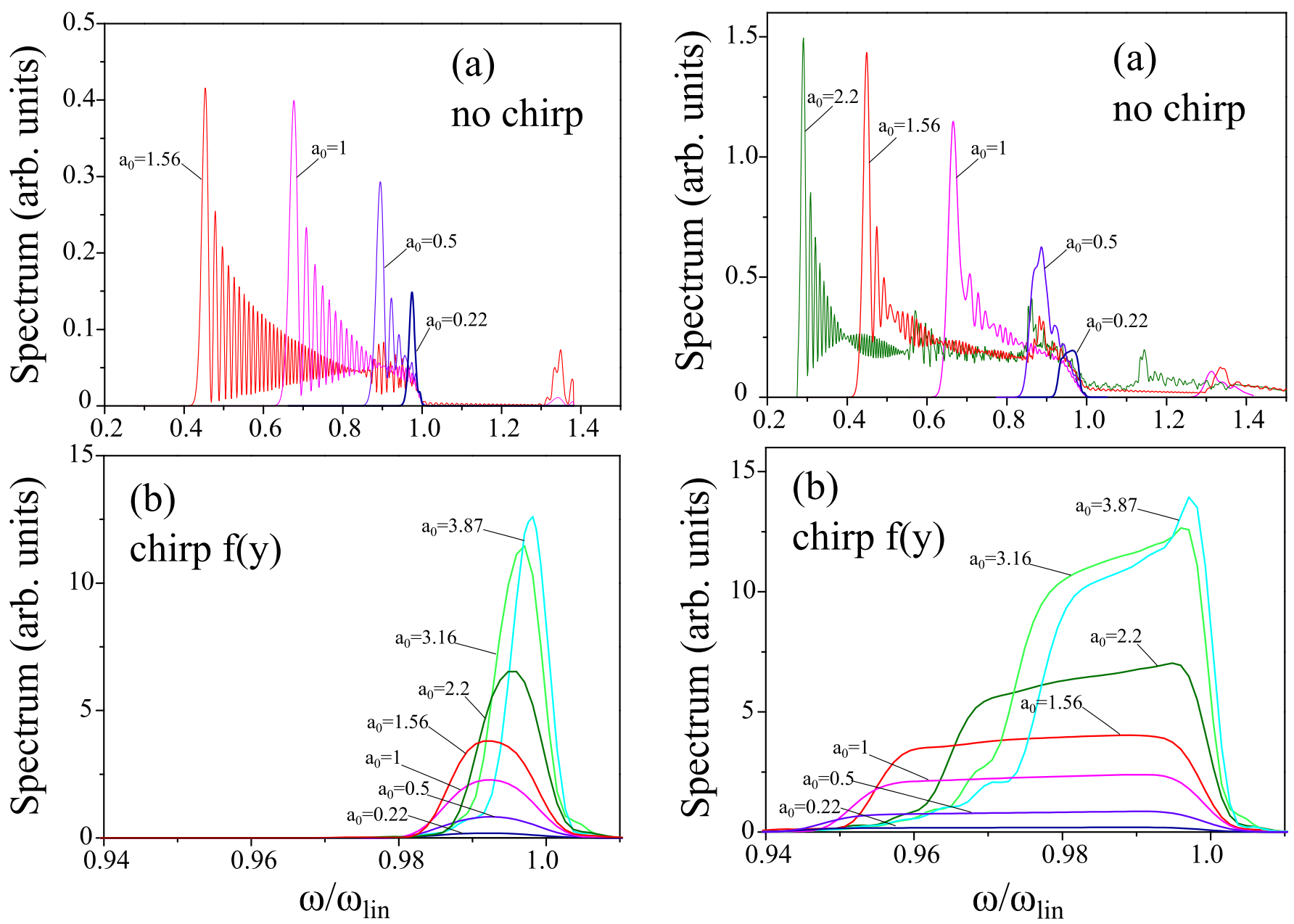

FIG. 6. Spectrum of the radiation collected within $\theta_{\max }=2 \mathrm{mrad}$, (a) without chirp, (b) with chirp $\mathrm{f}(\mathrm{y})$. The corresponding laser energies are: $\mathrm{E}=0.1 \mathrm{~J}$ $\left(a_{0}=0.22\right), \mathrm{E}=0.5 \mathrm{~J}\left(a_{0}=0.5\right), \mathrm{E}=2 \mathrm{~J}\left(a_{0}=1\right), \mathrm{E}=5 \mathrm{~J}\left(a_{0}=1.56\right)$, $\mathrm{E}=10 \mathrm{~J}\left(a_{0}=2.2\right), \mathrm{E}=20 \mathrm{~J}\left(a_{0}=3.16\right), \mathrm{E}=30 \mathrm{~J}\left(a_{0}=3.87\right)$.

The same cases, but with the chirp $f$, are presented in Figs. 6(b) and 7(b). Since the chirp has been chosen for compensating the broadening of the spectrum on axis, it acts less efficiently off-axis, and therefore a tail remains toward the low frequencies.

Figure 8 presents, at fixed value of $a_{0}$, the comparison of the spectrum on axis obtained with different values of $w_{0}$, for both chirp prescriptions $f_{0}$ [window (a)] and $f$ [window (b)]. For large values of $w_{0}$, the results are independent on the laser waist, and the plane wave model could be used. However, for decreasing $w_{0}$ (in this case $w_{0} \lesssim 15 \mu \mathrm{m}$ ), the gradients of the laser transverse distribution begin to affect the electron trajectories, and the chirp loses progressively efficiency. Contrarily to $f_{0}, f$ is able to correct the nonlinear broadening for more focused laser pulses, down to $w_{0}=5 \mu \mathrm{m}$. For smaller waists, the paraxial model is no more valid and a full wave integration of the Maxwell equations is needed.

Finally, in Fig. 9, we have compared, for the acceptance angles $\theta_{\max }=2 \mathrm{mrad}$ and $\theta_{\max }=4 \mathrm{mrad}$, the relative $\mathrm{rms}$ bandwidth $\Delta \omega / \omega$ on the fundamental frequency with and without chirp as a function of the laser parameter $a_{0}$.

In the case of a laser without chirp (empty stars), the radiation relative bandwidth strongly increases with $a_{0}$ due to the

FIG. 7. Spectrum of the radiation collected within $\theta_{\max }=4 \mathrm{mrad}$, (a) without chirp, (b) with chirp $\mathrm{f}(\mathrm{y})$. In (a), the effect of the harmonics produces the right tail.

non-linear broadening. In the case of a laser with chirp (full stars), instead, the relative bandwidth decreases from the linear prevision $(\triangle \omega / \omega)_{a c c+L, \theta_{\max }} \approx \sqrt{\left(\triangle \omega_{L} / \omega_{L}\right)^{2}+\left(\gamma_{0} \theta_{\max }\right)^{4} / 12}$ (green and dark green lines) to the laser bandwidth $\Delta \omega_{L} / \omega_{L}$ (yellow line). Even if the chirp is chosen in order to compensate the broadening on axis and is therefore less efficient off-axis, the bandwidth of the radiation collected within $\theta_{\max }=2 \mathrm{mrad}$ remains narrow due to the high and pronounced radiation intensity peak occurring in correspondence of the axis of the system. The present calculations are done in the case of a laser with Gaussian transverse and longitudinal profiles. The pulse is furthermore assumed transform limited. Deviations from this ideal case introduce other spectral broadenings with the effect of increasing the bandwidth.

\section{CONCLUSIONS}

We have derived a new expression of the chirp prescription that corrects the non-linear broadening on fundamental peak and harmonics up to laser pulse parameters well larger than 1, expanding and strengthening the result of Refs. 37 and 38. Realistic three dimensional situations are analyzed, 

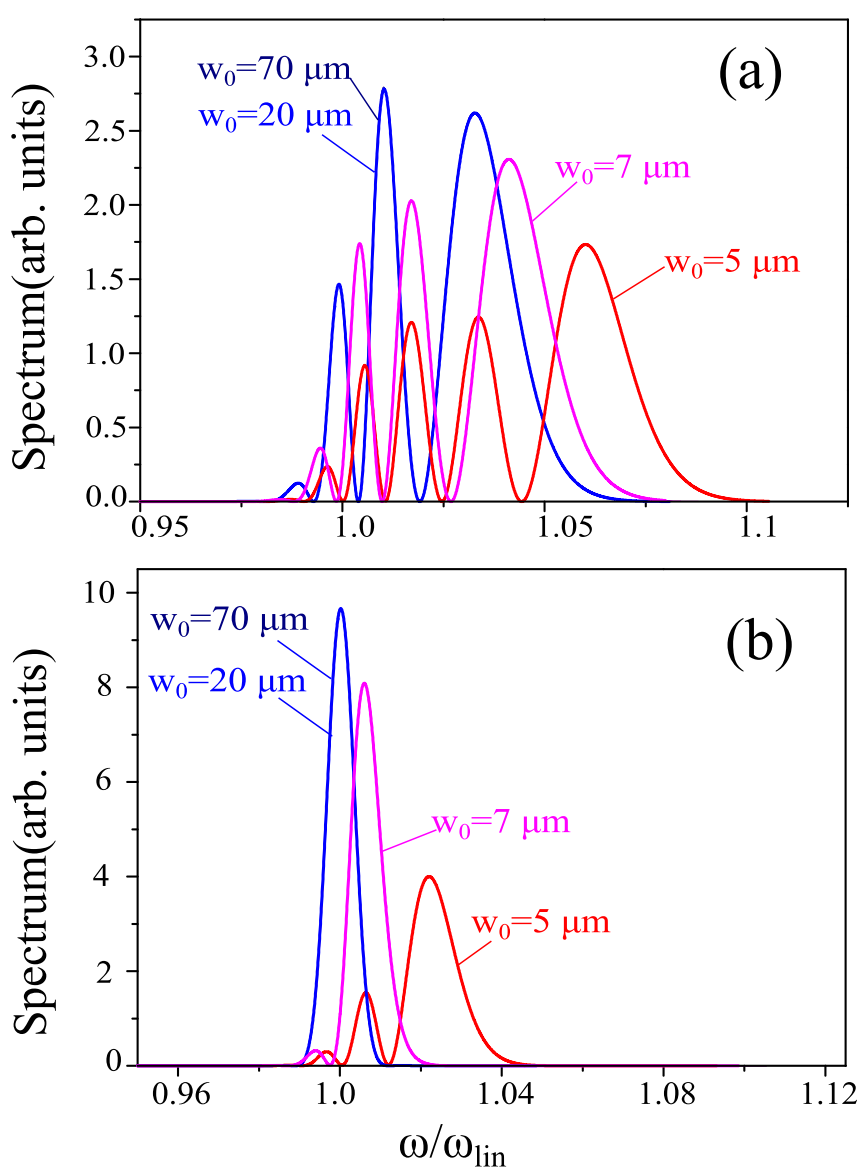

FIG. 8. Spectrum of the radiation on axis vs $\omega / \omega_{\text {lin }}$ for $a_{0}=0.6$ and $w_{0}$ $=70 \mu \mathrm{m}$ (blue line), $w_{0}=20 \mu \mathrm{m}$ (blue line), $w_{0}=7 \mu \mathrm{m}$ (magenta line), $w_{0}$ $=5 \mu \mathrm{m}$ (red line) for both chirp prescriptions $f_{0}$ [window (a)] and $f$ [window (b)].

using a focused laser pulse, collecting the chirped radiation within given acceptance angles and evaluating the spectrum. The robustness of the method will be therefore tested. The results demonstrate that increasing the laser parameter, the

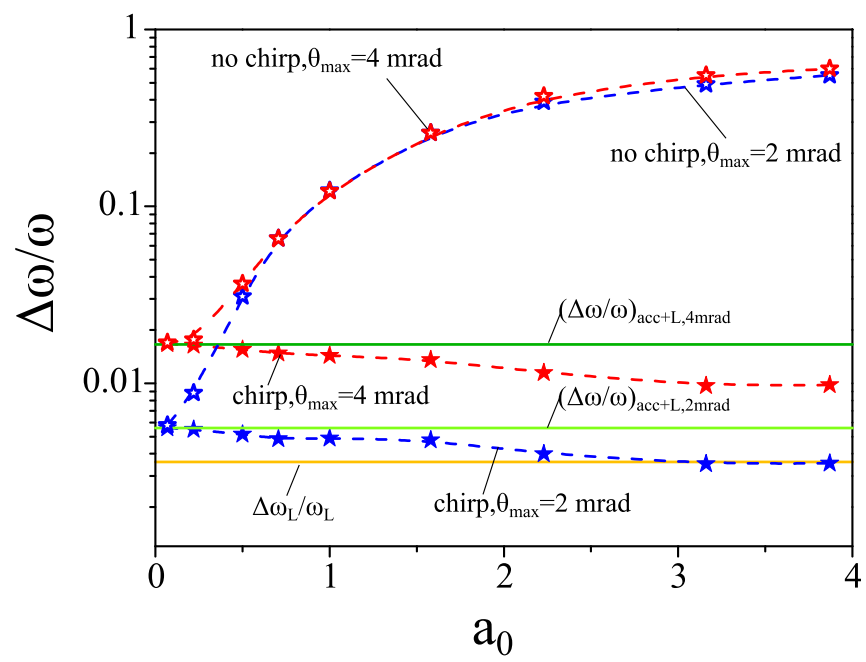

FIG. 9. Relative rms radiation bandwidth $\Delta \omega / \omega$ vs $a_{0}$ for $\theta_{\max }=2 \mathrm{mrad}$ (blue) and $\theta_{\max }=4 \mathrm{mrad}$ (red). Empty stars: without chirp. Full stars: with chirp $f$. On the plot, the laser bandwidth $\Delta \omega_{L} / \omega_{L}$ (yellow line) and the estimated linear bandwidths for the two acceptance angles, respectively, $(\Delta \omega)$ $\omega)_{a c c+L, 2 m r a d}$ (light green) and $(\Delta \omega / \omega)_{a c c+L, 2 m r a d}$ (dark green) are also shown. chirp permits one to decrease the rms bandwidth under the linear value, up to two orders of magnitude lower than the nonchirped case.

${ }^{1}$ C. M. Guenther, B. Pfau, R. Mitzner, B. Siemer et al., "Sequential femtosecond X-ray imaging," Nat. Photonics 5, 99 (2011).

${ }^{2}$ F. Tavella, N. Stojanovic, G. Geloni, and M. Gensch, "Few-femtosecond timing at fourth-generation X-ray light sources," Nat. Photonics 5, 162 (2011).

${ }^{3}$ I. V. Pogorelsky, I. Ben-Zvi, T. Hirose, S. Kashiwagi et al., "Demonstration of $8 \times 10^{18}$ photons/second peaked at $1.8 \AA$ in a relativistic Thomson scattering experiment," Phys. Rev. Spec. Top. Accel. Beams 3, 090702 (2000).

${ }^{4}$ W. Brown, S. Anderson, C. Barty, S. Betts, R. Booth, J. Crane et al., "Experimental characterization of an ultrafast Thomson scattering X-ray source with three-dimensional time and frequency-domain analysis," Phys. Rev. Spec. Top. Accel. Beams 7, 060702 (2004).

${ }^{5}$ M. Babzien, I. Ben-Zvi, K. Kusche, I. V. Pavlishin, I. V. Pogorelsky, D. P. Siddons, V. Yakimenko, D. Cline, F. Zhou, T. Hirose, Y. Kamiya, T. Kumita, T. Omori, J. Urakawa, and K. Yokoya, "Observation of the second harmonic in Thomson scattering from relativistic electrons," Phys. Rev. Lett. 96, 054802 (2006).

${ }^{6} \mathrm{M}$. Bech, O. Bunk, C. David, R. Ruth et al., "Hard X-ray phase-contrast imaging with the compact light source based on inverse Compton X-rays," J. Synchrotron Radiat. 16, 43 (2009).

${ }^{7}$ R. Kuroda, H. Toyokawa, M. Yasumoto, H. Ikeura-Sekiguchi, M. Koike, K. Yamada, T. Yanagida, T. Nakajyo, F. Sakai, and K. Mori, "Quasimonochromatic hard X-ray source via laser Compton scattering and its application," Nucl. Instrum. Methods Phys. Res. A 637, S183-S186 (2011).

${ }^{8}$ T. Akagi, A. Kosuge, S. Araki, R. Hajima, Y. Honda, T. Miyajima, M. Mori, R. Nagai, N. Nakamura, M. Shimada, T. Shizuma, N. Terunuma, and J. Urakawa, "Narrow-band photon beam via laser Compton scattering in an energy recovery linac," Phys. Rev. Accel. Beams 19, 114701 (2016). ${ }^{9}$ Y. Du, L. Yan, J. Hua, Q. Du, Z. Zhang, R. Li, H. Qian, W. Huang, H. Chen, and C. Tang, "Generation of first hard X-ray pulse at Tsinghua Thomson scattering X-ray source,” Rev. Sci. Instrum. 84, 053301 (2013).

${ }^{10}$ C. Vaccarezza et al., "The SPARC_LAB Thomson source," Nucl. Instrum. Methods Phys. Res. A 829, 237 (2016).

${ }^{11} \mathrm{E}$. Eggl et al., "X-ray phase-contrast tomosynthesis of a human ex vivo breast slice with an inverse Compton x-ray source," Europhys. Lett. 116, 68003 (2016).

${ }^{12}$ K. T. Phuoc, S. Corde, C. Thaury, V. Malka, A. Tafzi, J. P. Goddet, R. C. Shah, S. Sebban, and A. Rousse, "All-optical Compton gamma-ray source," Nat. Photonics 6, 308-311 (2012).

${ }^{13}$ N. D. Powers, I. Ghebregziabher, G. Golovin, C. Liu, S. Chen, S. Banerjee, J. Zhang, and D. P. Umstadter, "Quasi-monoenergetic and tunable X-rays from a laser-driven Compton light source," Nat. Photonics 8, 28-31 (2014).

${ }^{14}$ Y. Sakai et al., "Observation of redshifting and harmonic radiation in inverse Compton scattering," Phys. Rev. Spec. Top. Accel. Beams 18, 060702 (2015).

${ }^{15}$ B. Golosio, M. Endrizzi, P. Oliva, P. Delogu et al., "Measurement of an inverse Compton scattering source local spectrum using k-edge filters," Appl. Phys. Lett. 100, 164104 (2012).

${ }^{16}$ A. Jochmann, A. Irman, M. Bussmann, J. P. Couperus, T. E. Cowan, A. D. Debus, M. Kuntzsch, K. W. D. Ledingham, U. Lehnert, R. Sauerbrey, H. P. Schlenvoigt, D. Seipt, T. Stohlker, D. B. Thorn, S. Trotsenko, A. Wagner, and U. Schramm, "High resolution energy-angle correlation measurement of hard X rays from Laser-Thomson backscattering," Phys. Rev. Lett. 111, 114803 (2013).

${ }^{17}$ Y. Sakai et al., "Single shot, double differential spectral measurements of inverse Compton scattering in the nonlinear regime," Phys. Rev. Accel. Beams 20, 060701 (2017).

${ }^{18}$ H. Ikeura-Sekiguchi, R. Kuroda, M. Yasumoto, H. Toyokawa, M. Koike, K. Yamada, F. Sakai, K. Mori, K. Maruyama, H. Oka, and T. Kimata, "Inline phase-contrast imaging of a biological specimen using a compact laser-Compton scattering-based x-ray source," Appl. Phys. Lett. 92, 131107 (2008).

${ }^{19}$ K. Achterhold, M. Bech, S. Schleede, G. Potdevin, R. Ruth, R. Loewen, and F. Pfeiffer, "Monochromatic computed tomography with a compact laser-driven X-ray source," Sci. Rep. 3, 1313 (2013); S. Schleede, M. Bech, K. Achterhold et al., "Multimodal hard X-ray imaging of a 
mammography phantom at a compact synchrotron light source," J. Synchrotron Radiat. 19, 525-529 (2012).

${ }^{20}$ F. G. Meinel, F. Schwab, S. Schleede, M. Bech, J. Herzen, K. Achterhold, S. Auweter, F. Bamberg, A. O. Yildirim, A. Bohla, O. Eickelberg, R. Loewen, M. Gifford, R. Ruth, M. F. Reiser, F. Pfeiffer, and K. Nikolaou, "Diagnosing and mapping pulmonary emphysema on X-ray projection images: Incremental value of grating-based X-ray dark-field imaging," PLOS One 8, e59526 (2013); S. Schleede, F. G. Meinel, M. Bech et al., "Emphysema diagnosis using X-ray dark-field imaging at a laser-driven compact synchrotron light source," Proc. Natl. Acad. Sci. U. S. A. 109, 17880-17885 (2012)

${ }^{21}$ F. Schwab, S. Schleede, D. Hahn et al., "Comparison of contrast-to-noise ratios of transmission and dark-field signal in grating-based $\mathrm{X}$-ray imaging for healthy murine lung tissue," Z. Med. Phys. 23, 236-242 (2013).

${ }^{22} \mathrm{C}$. Sun and Y. K. Wu, "Theoretical and simulation studies of characteristics of a Compton light source," Phys. Rev. Spec. Top. Accel. Beams 14, 044701 (2011).

${ }^{23}$ A. Bacci et al., "Electron Linac design to drive bright Compton backscattering gamma-ray sources,” J. Appl. Phys. 113, 194508 (2013).

${ }^{24}$ V. Petrillo, A. Bacci, C. Curatolo, I. Drebot, A. Giribono, C. Maroli, A. R. Rossi, L. Serafini, P. Tomassini, C. Vaccarezza, and A. Variola, "Polarization of X-gamma radiation produced by a Thomson and Compton inverse scattering," Phys. Rev. Spec. Top. Accel. Beams 18, 110701 (2015).

${ }^{25}$ F. V. Hartemann, A. M. Tremaine, S. G. Anderson, C. P. J. Barty, S. M. Betts, R. Booth, W. J. Brown, J. K. Crane, R. R. Cross, D. J. Gibson, D. N. Fittinghoff, J. Kuba, G. P. Le Sage, D. R. Slaughter, A. J. Wootton, E. P. Hartouni, P. T. Springer, J. B. Rosenzweig, and A. K. Kerman, "Characterization of a bright, tunable, ultrafast Compton scattering X-ray source," Laser Part. Beams 22, 221-244 (2004).

${ }^{26}$ C. Curatolo, I. Drebot, V. Petrillo, and L. Serafini, "Analytical description of photon beam phase spaces in inverse Compton scattering sources," Phys. Rev. Spec. Top. Accel. Beams 20, 080701 (2017).

${ }^{27}$ N. Ranjan et al., "Simulation of inverse Compton scattering and its implications on the scattered linewidth," Phys. Rev. Accel. Beams 21, 030701 (2018).

${ }^{28}$ F. V. Hartemann, A. L. Troha, N. C. Luhmann, Jr., and Z. Toffano, "Spectral analysis of the nonlinear relativistic Doppler shift in ultrahigh intensity Compton scattering," Phys. Rev. E 54, 2956 (1996).

${ }^{29}$ G. A. Krafft, "Spectral distributions of Thomson-scattered photons from high-intensity pulsed lasers," Phys. Rev. Lett. 92, 204802 (2004).

${ }^{30} \mathrm{C}$. A. Brau, "Oscillations in the spectrum of nonlinear Thomson-backscattered radiation,” Phys. Rev. Spec. Top. Accel. Beams 7, 020701 (2004).
${ }^{31}$ F. He, Y. Y. Lau, D. P. Umstadter, and R. Kowalczyk, "Backscattering of an intense laser beam by an electron," Phys. Rev. Lett. 90, 055002 (2003).

${ }^{32}$ F. Albert, S. G. Anderson, D. J. Gibson, C. A. Hagmann, M. S. Johnson, M. Messerly, V. Semenov, M. Y. Shverdin, B. Rusnak, A. M. Tremaine, F. V. Hartemann, C. W. Siders, D. P. McNabb, and C. P. J. Barty, "Characterization and applications of a tunable, laser-based, MeV-class Compton-scattering $\gamma$-ray source," Phys. Rev. Spec. Top. Accel. Beams 13, 070704 (2010).

${ }^{33}$ F. V. Hartemann, F. Albert, C. W. Siders, and C. P. J. Barty, "Low-intensity nonlinear spectral effects in Compton scattering," Phys. Rev. Lett. 105, 130801 (2010).

${ }^{34}$ D. Seipt and B. Kampfer, "Scaling law for the photon spectral density in the nonlinear Thomson-Compton scattering," Phys. Rev. Spec. Top. Accel. Beams 14, 040704 (2011).

${ }^{35} \mathrm{M}$. Boca and V. Florescu, "Thomson and Compton scattering with an intense laser pulse,” Eur. Phys. J. D 61, 449 (2011).

${ }^{36}$ C. Maroli, V. Petrillo, P. Tomassini, and L. Serafini, "Nonlinear effects in Thomson backscattering," Phys. Rev. Spec. Top. Accel. Beams 16, 030706 (2013).

${ }^{37}$ B. Terzić, K. Deitrick, A. S. Hofler, and G. A. Krafft, "Narrow-band emission in Thomson sources operating in the high-field regime," Phys. Rev. Lett. 112, 074801 (2014).

${ }^{38}$ I. Ghebregziabher, B. A. Shadwick, and D. Umstadter, "Spectral bandwidth reduction of Thomson scattered light by pulse chirping," Phys. Rev. Spec. Top. Accel. Beams 16, 030705 (2013).

${ }^{39}$ A. Holkundkar, C. Harvey, and M. Marklund, "Thomson scattering in high-intensity chirped laser pulses," Phys. Plasmas 22, 103103 (2015).

${ }^{40}$ B. Terzić, C. Reeves, and G. A. Krafft, "Combining harmonic generation and laser chirping to achieve high spectral density in Compton sources," Phys. Rev. Spec. Top. Accel. Beams 19, 044403 (2016).

${ }^{41}$ S. G. Rykovanov, C. G. R. Geddes, C. B. Schroeder, E. Esarey, and W. P. Leemans, "Controlling the spectral shape of nonlinear Thomson scattering with proper laser chirping," Phys. Rev. Spec. Top. Accel. Beams 19, 030701 (2016).

${ }^{42}$ D. Seipt, S. G. Rykovanov, A. Surzhykov, and S. Fritzsche, "Narrowband inverse Compton scattering X-rays sources at high laser intensities," Phys. Rev. A 91, 033402 (2015).

${ }^{43}$ C. Harvey, M. Marklund, and A. R. Holkundkar, "Focusing effects in laser-electron Thomson scattering," Phys. Rev. Accel. Beams 19(9), 094701 (2016).

${ }^{44}$ J. D. Jackson, Classical Electrodynamics (John Wiley \& Sons, Inc., 1998). 